\title{
Rituximab in the treatment of B-cell non-Hodgkin lymphoma, focus on outcomes and comparative effectiveness
}

This article was published in the following Dove Press journal:

ClinicoEconomics and Outcomes Research

6 April 2010

Number of times this article has been viewed

\author{
Firas Badin \\ John Hayslip \\ University of Kentucky, Markey \\ Cancer Center, Lexington, KY, USA
}

Correspondence: John Hayslip

University of Kentucky, Markey Cancer

Center, 800 Rose St., CC450, Lexington,

KY 40536, USA

Email j.hayslip@uky.edu

\begin{abstract}
Rituximab is an important and well established component in the treatment of many patients with B-cell non-Hodgkin lymphoma. In this paper we review recent clinical trials investigating the addition of rituximab to standard chemotherapy regimens for treatment of patients with diffuse large B cell lymphoma and follicular lymphoma. This report focuses upon treatment efficacy, quality of life, and safety of rituximab or rituximab-containing regimens. More uniquely, we review economic aspects of lymphoma treatments, including the cost of standard chemotherapy regimens with or without rituximab, cost effectiveness of rituximab in both induction and maintenance treatment, and lymphoma's impacts on patient's productivity and their caregivers. We conclude that adding rituximab to standard chemotherapy treatment for patients with B-cell non-Hodgkin lymphoma is safe and cost-effective in numerous settings during both induction and maintenance therapies. Despite extensive review of the literature, many important questions have yet to be answered in the rituximab era and these represent important directions for future study.
\end{abstract}

Keywords: rituximab, lymphoma, cost effectiveness, transplant, safety

\section{Introduction}

Rituximab, an anti-CD20 monoclonal chimeric antibody, has significantly improved the prognosis of patients with B-cell non-Hodgkin lymphoma (NHL) and changed the economics of care delivery for these patients. Diffuse large B-cell lymphoma (DLBCL) is the most common histologic subtype of B-cell NHL accounting for approximately $25 \%$ of NHL cases. ${ }^{1,2}$ In 2005 the incidence rate of DLBCL in the United States was approximately 5 cases per 100,000 persons. Incidence varies by ethnicity with Caucasian Americans having the highest rates. Incidence increases with age; the median age at presentation is 64 years, and like most NHL there is a male predominance (male: female ratio 1.2:1). ${ }^{3}$ Follicular lymphoma (FL) is the second most common lymphoma in the western world accounting for more than $70 \%$ of indolent lymphomas and $22 \%$ of all NHL. ${ }^{3}$ It has 3 grades, grade I and II are indolent lymphomas while grade III is considered by many experts to be an aggressive lymphoma. It typically occurs in middle-aged or elderly adults with the median age at presentation of 60 years and a slight female predominance (male to female ratio $1: 1.4){ }^{3}$

\section{Rituximab}

Rituximab was the first widely adopted monoclonal antibody approved for cancer treatment. Rituximab is a monoclonal antibody directed against the CD20 antigen on B-lymphocytes. CD20 functions as a calcium channel important in B-cell survival. 
Rituximab's mechanism of action results from a combination of immune-mediated effects and possibly direct induction of apoptosis from binding to CD20. When rituximab binds to CD20 at the cell surface rituximab activates complementdependent cytotoxicity and human Fc receptors, mediating cell killing through an antibody-dependent cellular toxicity. As has been previously reviewed, the predominant mechanism of rituximab's anti-lymphoma activity is thought to be antibody-dependent cell-mediated cytotoxicity, with a lesser role for complement fixation. ${ }^{4}$ Although rituximab has multiple clinical uses, including autoimmune and rheumatologic disorders, this review focuses upon rituximab's use in B-cell NHL.

\section{DLBCL treatment}

The addition of rituximab concurrently with cytotoxic chemotherapy has improved the cure rates for patients with DLBCL (Table 1). Prior to the introduction of rituximab, SWOG-8516 (South Western Oncology Group) a randomized phase III trial of CHOP (cyclophosphamide, doxorubicin, vincristine, prednisone) vs m-BACOD (low-dose methotrexate with leucovorin rescue, bleomycin, doxorubicin, cyclophosphamide, vincristine, and dexamethasone) vs proMACE-CytaBOM (prednisone, doxorubicin, cyclophosphamide, and etoposide, followed by cytarabine, bleomycin, vincristine, and methotrexate with leucovorin rescue) vs MACOP-B (methotrexate with leucovorin rescue, doxorubicin, cyclophosphamide, vincristine, prednisone, and bleomycin) established that CHOP remained the standard chemotherapy for patients with advanced stage NHL demonstrating intermediate or higher grade histologic features. ${ }^{5}$ Although other regimens induced greater toxicities, no regimen showed an improvement in time to treatment failure or overall survival compared to the standard CHOP. ${ }^{5}$ The addition of rituximab to $\mathrm{CHOP}$ resulted in an approximately $10 \%$ absolute increase in survival beginning at one year from initiation of therapy in patients of all ages with minimal clinically relevant increases in toxicity. ${ }^{6,7}$ In the MabThera International Trial (MInT), 824 patients younger than 60 years with DLBCL (28\% stage III/IV and $48 \%$ with bulky disease) were randomly assigned to treatment with 6 cycles of CHOP-like chemotherapy with or without rituximab. ${ }^{8}$ Bulky and extra-nodal sites received additional radiotherapy. After a median follow-up of 34 months, patients assigned to Rituximab-CHOP (R-CHOP) had significantly higher rates of 3 -year event-free (79\% vs 59\%; $P<0.001)$ and overall (93\% vs 84\%; $P<0.0001)$ survival. In 3 randomized prospective studies consisting of approximately 2000 older patients ( $>65$ years of age) with advanced DLBCL, therapy with R-CHOP resulted in significantly higher overall survival at 3 (approximately $70 \%$ vs $57 \%$ for CHOP alone), 5 (58\% vs $45 \%$ ), and 7 years. ${ }^{9-13}$

Following these reports that the addition of rituximab to CHOP improved response rates and overall survival, further studies were conducted to investigate the impact of maintenance rituximab after initial chemotherapy. Patients who received CHOP chemotherapy for induction treatment have an initially improved survival when administered maintenance rituximab following CHOP chemotherapy compared to patients who received CHOP chemotherapy only. ${ }^{11}$ However, survival benefit disappeared with longer follow-up suggesting that unlike induction combination chemotherapy with rituximab, rituximab maintenance may delay clinically evident progression but does not increase the cure rate. ${ }^{14}$ Additionally, maintenance therapy with rituximab provided no significant benefit in those who received initial therapy with R-CHOP for DLBCL. ${ }^{14}$

Although the addition of rituximab to CHOP has improved the cure rates for patients with DLBCL,

Table I Treatment of diffuse large B-cell lymphoma

\begin{tabular}{|c|c|c|c|c|c|}
\hline Trial & Treatment & $\begin{array}{l}\text { Follow-up } \\
\text { period }\end{array}$ & Results & Schema & $P$ value \\
\hline$M \ln T^{8}$ & $\mathrm{CHOP}$ & 34 months & EFS: $59 \%$ OS: $79 \%$ & Ist line & EFS $P<0.001$ \\
\hline$N=824$ & $\mathrm{R}-\mathrm{CHOP}$ & & EFS: $84 \%$ OS: $93 \%$ & & OS $P<0.0001$ \\
\hline Habermann"l & $\mathrm{CHOP}$ & 3 years & FFS: $46 \%$ & Ist line & $P=0.04$ \\
\hline$N=415$ & $\mathrm{R}-\mathrm{CHOP}$ & & FFS: $53 \%$ & & \\
\hline Coiffier ${ }^{13}$ & $\mathrm{CHOP}$ & 7 years & EFS: $25 \%$ OS: $36 \%$ & Ist line & EFS $P<0.0001$ \\
\hline$N=399$ & $\mathrm{R}-\mathrm{CHOP}$ & & EFS: $42 \%$ OS: $53 \%$ & & OS $P=0.0004$ \\
\hline Kewalramani ${ }^{15}$ & ICE & 2 years & CRR: $27 \%$ PFS: $43 \%$ & 2nd line & PFS $P=0.25$ \\
\hline$N=36$ & R-ICE & & CRR: $53 \%$ PFS: $54 \%$ & & CRR $P=0.01$ \\
\hline
\end{tabular}

Abbreviations: EFS, event-free survival; OS, overall survival; FFS, failure-free survival; CRR, complete response rate; PFS, progression-free survival. 
a significant portion of patients still have recurrence and require additional therapies. Rituximab may play an important role in addition to standard chemotherapies in this setting as well. Thirty-six patients with relapsed or refractory DLBCL were treated with rituximab plus ifosfamide, carboplatin, and etoposide (R-ICE). ${ }^{15}$ The complete response rate was $53 \%$, significantly better than the historical control rate of $27 \%$ achieved for DLBCL treated with ICE alone $(P=0.01)$. Progression-free survival rates of patients who underwent transplantation after R-ICE trended toward improvement compared to historical controls who underwent transplantation after ICE (54\% vs $43 \%$ at 2 years) but weren't statistically significant in this analysis $(P=0.25)$. The ICE and R-ICE regimens have been very effective cytoreduction and stem cell mobilization regimens, and reasonable options for patients eligible for subsequent hematopoietic stem cell transplant (HSCT). ${ }^{15,16}$

Hematopoietic stem cell transplantation (HSCT) is a clinically necessary treatment for many patients with DLBCL and significantly impacts the economics of these patients' care. In the United States, autologous HSCT is considered standardof-care for many patients who achieve a second remission from DLBCL following a first recurrence. HSCT during the first remission is associated with significant morbidity and survival is similar in patients administered chemotherapy with or without HSCT. ${ }^{17}$ An intergroup trial (S9704) enrolled patients with high intermediate and high risk international prognostic index (IPI) scores according to age-adjusted index and randomized treatment to 6 cycles of R-CHOP followed by autologous HSCT vs 8 cycles of R-CHOP alone. We await results of this United States intergroup trial before recommending HSCT for this subgroup of patients.

Including rituximab with HSCT may improve the clinical results achieved with HSCT chemotherapy alone. A Phase III randomized trial (CORAL) comparing two rituximab-based regimens both followed by HSCT and maintenance rituximab or not in relapsed or refractory DLBCL found that rates of 2-year event-free survival were significantly reduced in a subset of patients with prior exposure to rituximab when compared with patients who were rituximab-naïve. ${ }^{18,19}$ In one modest-sized study, rituximab was given to 35 patients with recurrent or refractory aggressive NHL (25 with DLBCL) following high-dose therapy and autologous HSCT. ${ }^{20}$ Rituximab was given for 4 weeks starting at day 42 post-HSCT in all patients, and again at 6 months post-HSCT in 31 patients. At a median follow-up of 30 months, the estimated 2-year event-free and overall survivals were $81 \%$ and $85 \%$, respectively, for the 21 patients with relapsed or refractory DLBCL. Although delayed and severe neutropenia, including some patients with an absolute neutrophil count less than $500 / \mathrm{mm}^{3}$, as well as profound B-cell inhibition were observed, the treatment program was well-tolerated. In a second trial, high-dose rituximab, $1000 \mathrm{mg} / \mathrm{m}^{2}$ rather than standard-dose $375 \mathrm{mg} / \mathrm{m}^{2}$, was administered during stem cell mobilization, BEAM (carmustine, etoposide, cytarabine, melphalan) chemotherapy, and on days 1 and 8 after HSCT in 67 patients. ${ }^{21}$ At a median follow-up of 20 months, estimated 2-year disease-free and overall survival (OS) were $67 \%$ and $80 \%$, respectively ( $P=0.002)$, significantly better than those of a historical control group receiving the same preparative regimen without rituximab $43 \%$ and $53 \%$, respectively $(P=0.004)$.

\section{FL treatment}

Unlike DLBCL, no consensus exists for a standard-of-care initial treatment for patients with newly diagnosed FL. Indeed, the decision to begin treatment as opposed to continued observation is often a subjective decision. In the United States, significant regional variations exist in the care of patients with FL. For example, initial observation without treatment was recommended for $29 \%$ of patients in the northeast but only $13.3 \%$ of patients in the southeast. ${ }^{22}$ Cyclophosphamide, doxorubicin, and fludarabine based regimens may all be considered for first and subsequent treatments (Table 2).

The most commonly prescribed initial treatment for FL is R-CHOP, the same regimen that is considered standard-ofcare for DLBCL. ${ }^{22}$ The initial trial of R-CHOP for patients with de novo and recurrent FL reported a 95\% response rate and 55\% complete response rate. ${ }^{23}$ Subsequently, a randomized trial of CHOP vs R-CHOP for initial treatment of patients with FL reported a $90 \%$ response rate and $17 \%$ complete response rate for CHOP vs $96 \%$ and $20 \%$ respectively for R-CHOP $(P=0.011) .{ }^{24}$ For patients who do not receive $\mathrm{CHOP}$ for initial therapy, $\mathrm{CHOP}$ is often considered for second line therapy. As a second-line treatment, R-CHOP therapy has a significantly higher complete response rate than $\mathrm{CHOP}$ therapy alone, $30 \%$ vs $16 \%(P<0.0001)$, which translated into a significantly prolonged median progression-free survival (PFS) from first randomization, 33.1 vs 20.2 months $(P=0.0003) .{ }^{25}$

Another common FL treatment regimen, cyclophosphamide, vincristine, and prednisone (CVP) is significantly improved with the addition of rituximab. ${ }^{26}$ Adding rituximab to CVP (R-CVP) in previously untreated patients with stage III/IV FL improves complete response rates from 
Table 2 Treatment of follicular lymphoma

\begin{tabular}{|c|c|c|c|c|}
\hline Trial & Treatment & Results & Schema & $P$ value \\
\hline Hiddemann ${ }^{24}$ & $\mathrm{CHOP}$ & RR: $90 \%$ CR: $17 \%$ & Ist line & $P=0.011$ \\
\hline$N=428$ & $\mathrm{R}-\mathrm{CHOP}$ & RR: $96 \%$ CR: $20 \%$ & & \\
\hline van Oers ${ }^{25}$ & $\mathrm{CHOP}$ & ORR: $72 \%$ CR: I6\% & 2nd line & $P<0.001$ \\
\hline \multirow[t]{3}{*}{$N=465$} & $\mathrm{R}-\mathrm{CHOP}$ & ORR: $85 \%$ CR: $30 \%$ & & \\
\hline & RM & PFS: 52 mo OS: 85\% & 2nd line & PFS $P<0.001$ \\
\hline & OBS & PFS: 15 mo OS: $77 \%$ & & OS $P=0.011$ \\
\hline Marcus $^{26}$ & CVP & CRR: $57 \%$ OS: $77 \%$ & Ist line & CRR $P<0.0001$ \\
\hline$N=32 I$ & RCVP & CRR: $81 \%$ OS: $83 \%$ & & OS $P=0.029$ \\
\hline Forstpointner ${ }^{27}$ & FCM & ORR: $70 \%$ CR: $23 \%$ & Ist line & ORR $P=0.011$ \\
\hline$N=65$ & R-FCM & ORR: $94 \%$ CR: $40 \%$ & & \\
\hline Rummel $^{28}$ & $\mathrm{R}-\mathrm{CHOP}$ & ORR: $93 \%$ CR: $33 \%$ & Ist line & Not reported \\
\hline$N=437^{a}$ & $B R$ & ORR: $94 \%$ CR: 4 I\% & & \\
\hline Robinson ${ }^{29}$ & $B R$ & ORR: $92 \%$ CR: 4 I\% & 2nd line & $\mathrm{Cl} 95 \%$ \\
\hline$N=67^{b}$ & & & & \\
\hline
\end{tabular}

${ }^{2} 52 \%$ of the patients had follicular lymphoma, $20 \%$ had mantle cell lymphoma and $28 \%$ had other lymphoma.

${ }^{b}$ patients had indolent non-Hodgkin lymphoma and mantle cell lymphoma.

Abbreviations: $\mathrm{Cl}$, confidence interval; RM, rituximab maintenance; $\mathrm{OBS}$, observation; $\mathrm{BR}$, bendamustine with rituximab; mo, month; $\mathrm{RR}$, response rate; $\mathrm{CR}$, complete response; ORR, overall response rate; PFS, progression-free survival; OS, overall survival.

$57 \%$ to $81 \%$ and improves overall survival after 53 months median follow up from $77 \%$ to $83 \%$ without increasing serious adverse events $(P<0.0001)$.

Regimens containing fludarabine, a nucleoside analogue, have shown high response rates and are another considerable regimen for initial and subsequent therapy for patients with FL. Patients receiving R-FCM (rituximab, fludarabine, cyclophosphamide, mitoxantrone) as induction therapy will achieve significantly higher complete response (CR) and overall response rates (ORR) than those who receive induction with FCM alone. ${ }^{27}$ In a trial that combined patients with relapsed FL and mantle cell lymphoma and randomized patients to receive FCM with or without rituximab, the R-FCM arm was significantly superior in ORR, PFS and OS. In the FL subgroup, the patients receiving the rituximabcontaining induction arm had a 94\% ORR vs 70\% in FCM alone $\operatorname{arm}(P=0.011)$; PFS was also significantly longer in the R-FCM arm $(P=0.0139)$.

More recently, bendamustine and rituximab (BR) combinations have been studied in patients requiring initial treatment of FL and in patients with relapsed disease. In the frontline setting, BR has a similar overall and complete response rate $94 \%$ and $41 \%$ compared to R-CHOP $93 \%$ and $33 \%{ }^{28}$ The R-CHOP arm also had more hematologic and infectious toxicities than the BR arm. ${ }^{28}$ For patients with relapsed FL, BR has reported a $92 \%$ response rate with $41 \%$ complete response in a study of 67 patients. ${ }^{29}$ Increasingly,
$\mathrm{BR}$ is reasonable treatment option for initial or subsequent treatment for patients with FL.

Radioimmunotherapy, linkage of monoclonal antibody to radioisotope for intravenous administration, is another treatment option for patients with FL. In patients with advanced stage FL receiving $\mathrm{I}^{131}$-tositumomab as initial therapy, 95\% responded and $75 \%$ had a complete response. ${ }^{30}$ Radioimmunotherapy has also been investigated as a consolidation therapy after cytotoxic chemotherapy. An international, randomized, phase III trial evaluated the efficacy and safety of consolidation with Yttrium ${ }^{90}$ ibritumomab tiuxetan in patients with advanced-stage FL in first remission. Consolidation with $\mathrm{Y}^{90}$-ibritumomab tiuxetan is highly effective with no unexpected toxicities, prolonging PFS by 2 years and resulting in high partial response (PR) conversion to $\mathrm{CR}$ rates with multiple first-line induction combinations. ${ }^{31}$

Maintenance treatments with rituximab after patients are in remission from FL are effective at delaying time until recurrence in some instances for patients with FL. Two phase II trials of rituximab maintenance after rituximab monotherapy induction suggested that rituximab as a first-line treatment with scheduled maintenance at 6-month intervals produces high overall and complete response rates and a longer PFS , 34 months, than has been reported with a typical 4-week treatment alone. ${ }^{32,33}$ The Phase III Swiss Group for Clinical Cancer Research (SAKK) 35/98 trial enrolled both newly diagnosed and previously treated 
patients with FL. Overall, 151 patients (51 of whom were previously untreated) achieved CR, PR, or stable disease after rituximab monotherapy induction (four once weekly doses) and were subsequently randomized to either no further treatment or rituximab maintenance therapy consisting of four single rituximab infusions administered at 2-month intervals. In the initial publication, with a median follow-up of 35 months, median event-free survival among all patients receiving maintenance therapy was significantly longer than that achieved by patients receiving no further treatment, $23 \mathrm{vs}$ 12 months $(P=0.024) .{ }^{34}$ With 8 years of follow-up, no longterm toxicities were attributable to maintenance rituximab and $20 \%$ of patients still remained in remission. ${ }^{35}$ Overall this study showed that rituximab maintenance after rituximab monotherapy induction significantly improves outcomes in FL in terms of both response duration and event free survival, without causing additional toxicity. Recently, a comprehensive review and meta-analysis concluded that maintenance therapy with rituximab, either as 4-weekly infusions every 6 months or as a single infusion every 2 to 3 months, should be added to standard therapy for patients with relapsed or refractory FL after successful induction therapy. ${ }^{36}$

Rituximab maintenance after initial chemotherapy induction is significantly better than induction chemotherapy alone. A Phase III trial conducted by the Eastern Co-operative Oncology Group (ECOG 1496) studied 305 evaluable patients with newly diagnosed advanced stage indolent NHL. ${ }^{37}$ Most of these patients, $78 \%$, had advanced stage FL. Those patients achieving a complete or partial response or stable disease following CVP induction chemotherapy were randomized to either rituximab maintenance therapy or standard observation. The rituximab maintenance was dosed weekly for 4 doses and repeated at 6-month intervals for up to 2 years. Analyses conducted in the FL subgroup revealed 3-year PFS after random assignment was 64\% for the maintenance group vs 33\% for the observation group (hazard ratio $[\mathrm{HR}]=0.4 ; P<0.001$ ). Among patients with FL, OS at 3 years was $91 \%$ for the maintenance group vs $86 \%$ for the observation group ( $\mathrm{HR}=0.6 ; P=0.08)$. This study provides strong evidence that rituximab maintenance has a significant survival benefit for patients with FL who receive induction chemotherapy without the inclusion of rituximab during induction.

Similar, but more compelling, conclusions have been drawn in the relapsed setting. In European Organization for the Research and Treatment of Cancer (EORTC) protocol 20891, patients were randomized to $\mathrm{CHOP}$ or R-CHOP induction therapy for a first recurrence of FL. After six cycles of induction chemotherapy; patients who achieved complete or partial responses underwent another randomization to rituximab maintenance or standard observation. Rituximab maintenance significantly prolonged the PFS from 14.9 months in the observation to 51.5 months $(P<0.0001)$; rituximab maintenance also improved OS at 3 years from $77 \%$ with observation alone to $85 \% .{ }^{25}$ In a subgroup analysis of patients who received $\mathrm{CHOP}$ for induction, rituximab maintenance resulted in a median PFS from second randomization of 42.2 months vs 11.6 months in the observation $\operatorname{arm}(\mathrm{HR}, 0.30 ; P<0.001)$. For patients receiving $\mathrm{R}-\mathrm{CHOP}$ induction, rituximab maintenance resulted in a median PFS from second randomization 51.8 months vs 23.0 months in the observation arm $(\mathrm{HR}, 0.54 ; P=0.004)$. As a conclusion from this study; rituximab maintenance was still beneficial after R-CHOP induction but a survival benefit was clearer in patients who only received CHOP induction.

Maintenance rituximab after autologous HSCT is another interesting treatment consideration. The efficacy and safety of rituximab maintenance therapy administered once monthly after autologous HSCT were retrospectively analyzed in 27 patients with NHL treated at a single institution. ${ }^{38}$ Of these 27 patients, 12 had FL and were in CR at the time of transplantation. After a median follow-up period of 30 months, all 12 patients were still alive and, except for 1 patient who transformed from indolent to aggressive disease, there were no relapses. Another study incorporated in vivo purging with rituximab around the time of stem cell pheresis with maintenance rituximab after autologous HSCT. Twenty-three patients with relapsed FL were enrolled in this prospective single-arm study. ${ }^{39}$ Five-year OS and 5-year PFS are 78\% (95\% confidence interval [CI] 61\% to $95 \%$ ) and 59\% (95\% CI $38 \%$ to $80 \%$ ), respectively. Time to progression with the rituximab-containing regimen was significantly improved compared with each patient's previous treatment $(P<0.001)$. Durable molecular remissions, documented by quantitative polymerase chain reaction testing, occurred in 11 of 13 patients. Despite the prolonged hypogammaglobulinemia, no increase in major infections was observed.

\section{Cost of lymphomas}

The loss of life-years from DLBCL is hard to estimate and we found no previous reports in our review of the literature. A retrospective analysis of 374 patients with newly diagnosed stage II-IV aggressive NHL treated between 1993 and 2001 in The Netherlands with CHOP chemotherapy showed the mean first-line treatment costs (excluding G-CSF) were $€ 10,047$ (\$10,254) for patients younger than 60 years 
of age and $€ 12,232(\$ 12,484)$ for patients older than $60 .{ }^{40}$ Two-year follow-up costs averaged $€ 14,039$ (\$14,328) and $€ 9,026(\$ 9,211)$ for the two age groups, respectively. A large retrospective analysis of direct medical costs for the entire pathway of care for DLBCL was conducted between 1998 and 2004 in Canada. ${ }^{41}$ Patient samples were defined as those who received R-CHOP $(n=85)$ or CHOP $(n=86)$ as first-line treatment. CHOP cost was CAN\$12,240 $(\$ 8,800)$ and the 1 -year follow up cost was CAN\$8,929 $(\$ 6,400)$ compared to R-CHOP cost of CAN\$33,088 $(\$ 22,680)$ with 1-year follow up costs of CAN\$3,215 (\$2,311). In European healthcare systems between 2000 and 2003, the mean cumulative cost of CHOP was $\$ 3,358$ and R-CHOP was $\$ 17,225 .{ }^{42}$ The posttreatment cancer surveillance cost for $\mathrm{CHOP}$ was $\$ 3,950$ compared with \$5,202 for R-CHOP.

Often thought of as one of the most costly treatments, HSCT adds significant expense to the care of patients who require this modality of care. For patients with refractory or relapsed NHL, the cost from induction chemotherapy until 3 months after discharge from inpatient care following the transplant was $€ 15,000$ (\$15,300) from 1994 to 1998 in The Netherlands. ${ }^{43}$ Similarly, the cost for autologous HSCT for the Canadian system in 1993/1994 was CAN\$ 22,089 $(\$ 16,029){ }^{44}$

The loss of life-years is harder to estimate from FL because patients may undergo multiple series of treatments over a decade or more after diagnoses. Although the attempts to capture the costs of chemotherapy for FL have not always accounted for infusion related and follow up costs, in 2007 the cost of 8 cycles of CVP in the US was $\$ 500,6$ cycles R-CVP \$24,500, and CHOP as salvage over 6 months costs $\$ 3,829 .{ }^{45}$ Further, autologous HSCT for treatment of FL in the US in 2007 was estimated to cost $\$ 75,352 .{ }^{45}$ Although this cost is much higher than reviewed for DLBCL, this difference is likely related to healthcare inflation and differences in cost between healthcare systems and not due to differences between the diseases. As with DLBCL, the cost to patients and families for lost productivity is likely significant, but has not been well measured.

Despite the extensive publications about NHL treatments, little is known about how treatment impacts on patients' work and daily activities. A cross-sectional study of work productivity, activity impairment, and impacts on caregivers was conducted with 84 patients with NHL. Of the $71 \%$ of patients who worked before diagnosis; only $41 \%$ continue to work after treatment with $36 \%$ transitioning to retirement, sick leave (10\%) and unemployment (4\%). ${ }^{46}$ Active chemotherapy treatment was associated with significant activity impairment
(OR $14.5 ; 95 \%$ CI 0.91 to $230.9 ; P=0.059$ ). A significant proportion of patients required caregiver assistance (23\%); with $33 \%$ of their working days being missed as a consequence of this care. ${ }^{46}$ Those caregivers suffered from physical, psychological, and financial disruptions. Another large cohort study reported that $13 \%$ of all survivors had stopped working for cancer-related reasons within 4 years of diagnosis. More than $50 \%$ of all survivors had stopped working after the first year with $75 \%$ of those who stopped working returning to work in the future when they are off treatment. ${ }^{47}$ Survivors of stage IV blood and lymph malignancies have one of the highest adjusted risk of disability or quitting work amongst all patients with cancer. ${ }^{47}$

Given the high costs of these illnesses and their treatments; much work has gone into determining the most cost-effective approaches for care delivery (Table 3). A cost-effectiveness analysis of $\mathrm{CHOP}$ vs R-CHOP for initial treatment of patients with DLBCL has been presented from a European payer perspective. ${ }^{48}$ Chemotherapy cost was estimated from a phase III trial in France, Belgium, and Switzerland. The survival and cost-effectiveness was estimated from 4 years to 15 years. R-CHOP resulted in $20.6 \%$ relative increase in complete response rate, absolute increase from $63 \%$ to $76 \%$, and a $31 \%$ decrease in risk of death at four years. Over 15 years mean, median OS was estimated to be 6.9 years for R-CHOP and 5.74 years for CHOP, a mean increase in OS of 1.16 years (or 1.07 quality adjusted life year [QALY]s). Total direct medical costs were $€ 13,170$ (\$11,250) higher with R-CHOP, with an incremental cost-effectiveness ratio of $€ 12,259(\$ 10,477)$ per QALY gained which looks favorable comparing to standard worldwide accepted ranges. Similarly, the incremental cost effectiveness of CHOP vs R-CHOP in DLBCL patients in Netherlands has been reported. ${ }^{49} \mathrm{~A}$ transition state model was developed to estimate the clinical course, costs and quality

Table 3 Cost of diffuse large B-cell lymphoma treatment

\begin{tabular}{|c|c|c|c|c|}
\hline \multirow[t]{2}{*}{ Study } & \multirow[t]{2}{*}{ Treatment } & \multirow{2}{*}{$\begin{array}{l}\text { Follow-up } \\
\text { period }\end{array}$} & \multicolumn{2}{|l|}{ Cost } \\
\hline & & & $\begin{array}{l}\text { Ist line } \\
\text { cost }\end{array}$ & $\begin{array}{l}\text { Follow-up } \\
\text { cost }\end{array}$ \\
\hline Hornberger ${ }^{42}$ & $\mathrm{CHOP}$ & 5 years & $\$ 3,358$ & $\$ 3,950$ \\
\hline$N=399$ & $\mathrm{R}-\mathrm{CHOP}$ & & $\$ 17,225$ & $\$ 5,202$ \\
\hline $\begin{array}{l}\text { van Agthoven }{ }^{43} \\
N=374\end{array}$ & $\mathrm{CHOP}$ & 2 years & $\begin{array}{l}\$ 10,254 \\
\$ 12,232\end{array}$ & $\begin{array}{l}\$ 9,211- \\
\$ 14,328\end{array}$ \\
\hline $\operatorname{Lee}^{4 l}$ & $\mathrm{CHOP}$ & I year & $\$ 8,800$ & $\$ 6,400$ \\
\hline$N=|7|$ & $\mathrm{R}-\mathrm{CHOP}$ & & $\$ 22,680$ & $\$ 2,311$ \\
\hline
\end{tabular}


of life (QOL) differences between the two groups. The only costs included were direct medical costs. The time horizon of the model was 15 years. The incremental gain was 0.88 QALYs favoring the addition of rituximab to CHOP. The costs were $€ 12,343(\$ 10,550)$ higher in the younger group of patients and $€ 15,860(\$ 13,555)$ in the older patients. ${ }^{49}$ This resulted in an incremental cost-effectiveness ratio (ICER) of $€ 13,983(\$ 11,950)$ for the younger and $€ 17,933(\$ 15,327)$ for the older patients per QALY gained. ${ }^{49}$ An additional study compared the direct medical cost of CHOP and R-CHOP in young patients with favorable IPI risk DLBCL from the perspective of the Italian National Health Service. ${ }^{50}$ The model provided an estimate of mean survival and mean costs over a 3-year period. The QALYs gained with R-CHOP was greater than with CHOP. In the R-CHOP regimen, the additional costs of rituximab $€ 10,086(\$ 14,780)$ were balanced by the lower additional therapy costs $€ 10,803$ (\$15830) leading to a lower overall mean treatment cost per patient with R-CHOP regimen than with the $\mathrm{CHOP}$ regimen $€ 22,113(\$ 32,400)$ vs $€ 22,831$ ( $\$ 33,460)$.

In FL, the cost effectiveness of adding rituximab to CVP has been reported ${ }^{45}$ and the mean overall survival is projected to be 1.51 years longer for patients receiving R-CVP than CVP. The cost per QALY gained is $\$ 28,565$ and the ICER of R-CVP compared with CVP is projected to be acceptable under a range of sensitivity analyses in the United States. The cost-effectiveness of maintenance rituximab for US patients with FL in second remission has also been reported. ${ }^{51}$ Five years after R-CHOP and achieving a second remission, disease-free survival is expected to be $47 \%$ and $22 \%$, and the OS rates are estimated to be $73 \%$ and $61 \%$ for extended adjuvant rituximab and observation, respectively. The discounted ICER for the addition of adjuvant rituximab is estimated to be $\$ 19,522$ per QALYs gained. The authors concluded that maintenance rituximab offers a clinical benefit to patients who have a second remission from FL at a cost generally acceptable to the US healthcare system. Similarly, a cost-effectiveness analysis of maintenance rituximab during second remission for patients in Sweden was conducted and concluded to be a reasonable value for patients who achieve a complete response to induction chemotherapy. ${ }^{52}$

\section{Patient focused outcomes}

The addition of rituximab to CHOP chemotherapy may not add significantly to the symptoms or toxicities of CHOP chemotherapy alone. QOL was evaluated during a prospective randomized trial of rituximab maintenance therapy in 91 NHL patients (38 with DLBCL and 16 with FL).$^{53}$
QOL was assessed with the standardized questionnaires EORTC-QLQ-C30, EuroQol-5D, and EuroQol-5D (VAS). No differences in QOL were found between the groups that received rituximab maintenance or standard observation. We are not aware of any reports that have specifically ascertained patient satisfaction with receiving rituximab during treatment for DLBCL or adherence with treatment recommendations. Similarly, uptake of R-CHOP in place of CHOP seems nearly universal when health care systems are able to offer rituximab, although objective data to support this belief are lacking.

In FL, the addition of rituximab to $\mathrm{CHOP}$ did not clinically significantly increase the toxicity of induction therapy. ${ }^{25}$ In SAKK 35/98, maintenance rituximab was well tolerated. Of the 137 patients who were evaluable for toxicity, only $7 \%$ in both the maintenance and observation arms reported toxicities. ${ }^{34} \mathrm{In}$ Eastern Cooperative Oncology Group (ECOG) protocol 1496, rituximab maintenance or observation after induction therapy with CVP, rituximab maintenance was well tolerated and did not lead to significantly higher rates of neutropenia, thrombocytopenia, or infection compared with observation alone. ${ }^{37}$ In EORTC 20891, rituximab maintenance vs observation after induction therapy with either CHOP or R-CHOP, rituximab maintenance treatment was associated with minimal toxicity. ${ }^{25}$ Only 6 out of 167 patients (4\%) had to discontinue treatment because of toxicity. Four of the six patients were discontinued due to infections, and there was no treatment-related mortality. As in DLBCL, patient satisfaction, acceptability, physician uptake, and adherence have been widely reported and our literature review failed to return any relevant reports.

\section{Future directions}

Although there are many investigations of rituximab in NHL many questions still await an answer. Despite the fact that all studied maintenance schedules have been shown to be effective in FL; we are still unsure about the optimal dosing, schedule, and duration of this maintenance. Because all of the rituximab maintenance studies have been conducted over the last 7 years, there is no clear guidance yet about long term safety and efficacy. Questions remain about the risks of decreased immunoglobulin levels and infection rates, activating cutaneous squamous cell carcinoma, and selection for CD20 negative lymphoma relapses. Questions about the long-term safety require longer follow up. The impact of rituximab maintenance on patient's QOL and productivity and caregiver burden should be studied further. Some researchers and advocates have suggested that receiving maintenance treatments may reduce QOL due to treatment 
burden but others have advocated that maintenance may increase patient's feeling of satisfaction as they participate in actively managing their disease. Further research is clearly indicated. Despite our careful review, we are not aware of data to assess patients' acceptance or physicians' uptake of rituximab. Although treatment with rituximab is clinically beneficial in several patient settings, future investigations may allow doctors and patients to optimize the treatment effect and refine approaches that improve patient-focused outcomes and limit economic burden.

\section{Disclosures}

The authors report no conflicts of interest.

\section{References}

1. Swerdlow SH, Campo E, Harris NL, et al. World Health Organization Classification of Tumours of Haematopoietic and Lymphoid Tissues. 4th Edition. Lyon France: IARC Press; 2008.

2. Morton LM, Wang SS, Devesa SS, Hartge P, Weisenburger DD, Linet MS. Lymphoma incidence patterns by WHO subtype in the United States, 1992-2001. Blood. 2006;107(1):265-276.

3. A clinical evaluation of the International Lymphoma Study Group classification of non-Hodgkin's lymphoma. The non-Hodgkin's lymphoma Classification Project. Blood. 1997;89(11):3909-3918.

4. Maloney DG, Smith B, Rose A. Rituximab: mechanism of action and resistance. Semin Oncol. 2002;29(1 Suppl 2):2-9.

5. Fisher RI, Gaynor ER, Dahlberg S, et al. Comparison of a standard regimen (CHOP) with three intensive chemotherapy regimens for advanced non-Hodgkin's lymphoma. N Engl J Med. 1993;328(14): 1002-1006.

6. Pettengell R, Linch D. Position paper on the therapeutic use of rituximab in CD20-positive diffuse large B-cell non-Hodgkin's lymphoma. Br J Haematol. 2003;121(1):44-48.

7. Sehn LH, Donaldson J, Chhanabhai M, et al. Introduction of combined CHOP plus rituximab therapy dramatically improved outcome of diffuse large B-cell lymphoma in British Columbia. J Clin Oncol. 2005;23(22):5027-5033.

8. Pfreundschuh M, Trumper L, Osterborg A, et al. CHOP-like chemotherapy plus rituximab versus CHOP-like chemotherapy alone in young patients with good-prognosis diffuse large-B-cell lymphoma: a randomised controlled trial by the MabThera International Trial (MInT) Group. Lancet Oncol. 2006;7(5):379-391.

9. Coiffier B, Lepage E, Briere J, et al. CHOP chemotherapy plus rituximab compared with CHOP alone in elderly patients with diffuse large-B-cell lymphoma. $N$ Engl J Med. 2002;346(4):235-242.

10. Feugier P, Van Hoof A, Sebban C, et al. Long-term results of the R-CHOP study in the treatment of elderly patients with diffuse large B-cell lymphoma: a study by the Groupe d'Etude des Lymphomes de l'Adulte. J Clin Oncol. 2005;23(18):4117-4126.

11. Habermann TM, Weller EA, Morrison VA, et al. Rituximab-CHOP versus CHOP alone or with maintenance rituximab in older patients with diffuse large B-cell lymphoma. J Clin Oncol. 2006;24(19): 3121-3127.

12. Pfreundschuh M, Schubert J, Ziepert M, et al. Six versus eight cycles of bi-weekly CHOP-14 with or without rituximab in elderly patients with aggressive CD20+ B-cell lymphomas: a randomised controlled trial (RICOVER-60). Lancet Oncol. 2008;9(2):105-116.

13 Coiffier B, Feugier P, Mounier, et al. Long-term results of the GELA study comparing R-CHOP and CHOP chemotherapy in older patients with diffuse large B-cell lymphoma show good survival in poor-risk patients. J Clin Oncol. (Meeting Abstracts) 2007;25(18 suppl):8009.
14 Morrison VA, Weller EA, Habermann, et al. Maintenance rituximab (MR) compared to observation (OBS) after R-CHOP or CHOP in older patients (pts) with diffuse large B-cell lymphoma (DLBCL): An Intergroup E4494/C9793 update. J Clin Oncol. (Meeting Abstracts) 2007;25(18_suppl):8011.

15. Kewalramani T, Zelenetz AD, Nimer SD, et al. Rituximab and ICE as second-line therapy before autologous stem cell transplantation for relapsed or primary refractory diffuse large B-cell lymphoma. Blood. 2004;103(10):3684-3688.

16. Hertzberg M, Crombie C, Benson W, et al. Outpatient fractionated ifosfamide, carboplatin, and etoposide as salvage therapy in relapsed and refractory non-Hodgkin's and hodgkin's lymphoma. Ann Oncol. 2006; 17 :iv25.

17. Greb A, Bohlius J, Schiefer D, Schwarzer G, Schulz H, Engert A. Highdose chemotherapy with autologous stem cell transplantation in the first line treatment of aggressive non-Hodgkin lymphoma (NHL) in adults. Cochrane Database Syst Rev. 2008;(1):CD004024.

18. Gisselbrecht C, Schmitz N, Mounier N, et al. R-ICE versus R-DHAP in relapsed patietns with CD20 diffuse large B-cell lymphoma followed by stem cell transplantation and maintenance treatment with rituximab or not: First interim analysis of 200 patients. Blood. 2007;110(11):517.

19. Gisselbrecht C, Glass B, et al. R-ICE versus R-DHAP in relapsed patients with CD20 diffuse large B-cell lymphoma (DLBCL) followed by autologous stem cell transplantation: CORAL study. J Clin Oncol. 2009;27(15S):8509.

20. Horwitz SM, Negrin RS, Blume KG, et al. Rituximab as adjuvant to high-dose therapy and autologous hematopoietic cell transplantation for aggressive non-Hodgkin lymphoma. Blood. 2004;103(3):777-783.

21. Khouri IF, Saliba RM, Hosing C, et al. Concurrent administration of high-dose rituximab before and after autologous stem-cell transplantation for relapsed aggressive B-cell non-Hodgkin's lymphomas. J Clin Oncol. 2005;23(10):2240-2247.

22. Friedberg JW, Taylor MD, Cerhan JR, et al. Follicular lymphoma in the United States: first report of the national LymphoCare study. J Clin Oncol. 2009;27(8):1202-1208.

23. Czuczman MS, Grillo-Lopez AJ, White CA, et al. Treatment of patients with low-grade B-cell lymphoma with the combination of chimeric antiCD20 monoclonal antibody and CHOP chemotherapy. J Clin Oncol. 1999;17(1):268-276.

24. Hiddemann W, Kneba M, Dreyling M, et al; Frontline therapy with rituximab added to the combination of cyclophosphamide, doxorubicin, vincristine, and prednisone (CHOP) significantly improves the outcome for patients with advanced-stage follicular lymphoma compared with therapy with CHOP alone: results of a prospective randomized study of the German Low-Grade Lymphoma Study Group. Blood. 2005;106(12):3725-3732.

25. van Oers $M H$, Klasa R, Marcus RE, et al. Rituximab maintenance improves clinical outcome of relapsed/resistant follicular non-Hodgkin lymphoma in patients both with and without rituximab during induction: results of a prospective randomized phase 3 intergroup trial. Blood. 2006;108(10):3295-3301.

26. Marcus R, Imrie K, Solal-Celigny P, et al. Phase III study of R-CVP compared with cyclophosphamide, vincristine, and prednisone alone in patients with previously untreated advanced follicular lymphoma. J Clin Oncol. 2008;26(28):4579-4586.

27. Forstpointner R, Dreyling M, Repp R, et al; The addition of rituximab to a combination of fludarabine, cyclophosphamide, mitoxantrone (FCM) significantly increases the response rate and prolongs survival as compared with FCM alone in patients with relapsed and refractory follicular and mantle cell lymphomas: results of a prospective randomized study of the German Low-Grade Lymphoma Study Group. Blood. 2004;104(10):3064-3071.

28 Rummel MJ, von Gruenhagen U, Niederle N, et al. Bendamustine plus rituximab versus CHOP plus rituximab in the first-line-treatment of patients with follicular, indolent and mantle cell lymphomas: results of a randomized phase III study of the Study Group Indolent Lymphomas (StiL). Blood. (ASH Annual Meeting Abstracts) 2008;112(11):2596. 
29. Robinson KS, Williams ME, van der Jagt RH, et al. Phase II multicenter study of bendamustine plus rituximab in patients with relapsed indolent B-cell and mantle cell non-Hodgkin's lymphoma. J Clin Oncol. 2008;26(27):4473-4479.

30. Kaminski MS, Tuck M, Estes J, et al. 131I-tositumomab therapy as initial treatment for follicular lymphoma. N Engl J Med. 2005;352(5):441-449.

31. Morschhauser F, Radford J, Van Hoof A, et al. Phase III trial of consolidation therapy with yttrium-90-ibritumomab tiuxetan compared with no additional therapy after first remission in advanced follicular lymphoma. J Clin Oncol. 2008;26(32):5156-5164.

32. Hainsworth JD, Litchy S, Burris HA 3rd, et al. Rituximab as first-line and maintenance therapy for patients with indolent non-Hodgkin's lymphoma. J Clin Oncol. 2002;20(20):4261-4267.

33. Hainsworth JD, Litchy S, Morrissey L, Yardley DA, Greco FA. Rituximab as frontline and maintenance therapy for indolent non-Hodgkin's lymphoma (NHL): longterm Follow-up of a Minnie Pearl Cancer Research Network Phase II trial. Blood. 2003;102:1a-2a.

34. Ghielmini M, Schmitz SF, Cogliatti SB, et al. Prolonged treatment with rituximab in patients with follicular lymphoma significantly increases event-free survival and response duration compared with the standard weekly $\times 4$ schedule. Blood. 2004;103(12):4416-4423.

35. Ghielmini M, Hsu Smitz S, Al e. Long-term follow-up of patients with follicular lymphoma (FL) receiving single agent rituximab at two different schedules in study SAKK 35/98. J Clin Oncol. 2009;27(15S):8512.

36. Vidal L, Gafter-Gvili A, Leibovici L, Shpilberg O. Rituximab as maintenance therapy for patients with follicular lymphoma. Cochrane Database Syst Rev. 2009;(2):CD006552.

37. Hochster H, Weller E, Gascoyne RD, et al. Maintenance rituximab after cyclophosphamide, vincristine, and prednisone prolongs progressionfree survival in advanced indolent lymphoma: results of the randomized phase III ECOG1496 Study. J Clin Oncol. 2009;27(10):1607-1614

38. Neumann F, Harmsen S, Martin S, et al. Rituximab long-term maintenance therapy after autologous stem cell transplantation in patients with B-cell non-Hodgkin's lymphoma. Ann Hematol. 2006 Aug;85(8): $530-534$

39. Hicks LK, Woods A, Buckstein R, et al. Rituximab purging and maintenance combined with auto-SCT: long-term molecular remissions and prolonged hypogammaglobulinemia in relapsed follicular lymphoma. Bone Marrow Transplant. 2009;43(9):701-708.

40. van Agthoven M, Sonneveld P, Verdonck LF, Uyl-de Groot CA. Cost determinants in aggressive non-Hodgkin's lymphoma. Haematologica. 2005;90(5):661-671.

41. Lee RC, Zou D, Demetrick DJ, Difrancesco LM, Fassbender K, Stewart D. Costs associated with diffuse large B-cell lymphoma patient treatment in a Canadian integrated cancer care center. Value Health. 2008;11(2):221-230.
42. Hornberger JC, Best JH. Cost utility in the United States of rituximab plus cyclophosphamide, doxorubicin, vincristine, and prednisone for the treatment of elderly patients with diffuse large B-cell lymphoma. Cancer. 2005;103(8):1644-1651.

43. van Agthoven M, Vellenga E, Fibbe WE, Kingma T, Uyl-de Groot CA. Cost analysis and quality of life assessment comparing patients undergoing autologous peripheral blood stem cell transplantation or autologous bone marrow transplantation for refractory or relapsed non-Hodgkin's lymphoma or Hodgkin's disease. a prospective randomised trial. Eur J Cancer. 2001;37(14):1781-1789.

44. Bredeson C, Malcolm J, Davis M, Bence-Bruckler I, Kearns B, Huebsch L. Cost analysis of the introduction of PBPC for autologous transplantation: effect of switching from bone marrow (BM) to peripheral blood progenitor cells (PBPC). Bone Marrow Transplant. 1997;20(10): 889-896.

45. Hornberger J, Reyes C, Lubeck D, Valente N. Economic evaluation of rituximab plus cyclophosphamide, vincristine and prednisolone for advanced follicular lymphoma. Leuk Lymphoma. 2008;49(2): $227-236$.

46. Cheung MC, Imrie KR, Friedlich J, Buckstein R, Lathia N, Mittmann N. The impact of follicular (FL) and other indolent non-Hodgkin's lymphomas (NHL) on work productivity-a preliminary analysis. Psychooncology. 2009;18(5):554-559.

47. Short PF, Vasey JJ, Tunceli K. Employment pathways in a large cohort of adult cancer survivors. Cancer. 2005 Mar 15;103(6):1292-1301.

48. Best JH, Hornberger J, Proctor SJ, Omnes LF, Jost F. Cost-effectiveness analysis of rituximab combined with $\mathrm{CHOP}$ for treatment of diffuse large B-cell lymphoma. Value Health. 2005;8(4):462-470.

49. Groot MT, Lugtenburg PJ, Hornberger J, Huijgens PC, Uyl-de Groot CA. Cost-effectiveness of rituximab (MabThera) in diffuse large B-cell lymphoma in The Netherlands. Eur J Haematol. 2005;74(3): 194-202.

50. Ferrara F, Ravasio R. Cost-effectiveness analysis of the addition of rituximab to CHOP in young patients with good-prognosis diffuse large-B-cell lymphoma. Clin Drug Investig. 2008;28(1):55-65.

51. Hayslip JW, Simpson KN. Cost-effectiveness of extended adjuvant rituximab for US patients aged 65-70 years with follicular lymphoma in second remission. Clin Lymphoma Myeloma. 2008 Jun;8(3): $166-170$.

52. Kasteng F, Erlanson M, Hagberg H, Kimby E, Relander T, Lundkvist J. Cost-effectiveness of maintenance rituximab treatment after second line therapy in patients with follicular lymphoma in Sweden. Acta Oncol. 2008;47(6):1029-1036.

53. Witzens-Harig M, Reiz M, Heiss C, et al. Quality of life during maintenance therapy with the anti-CD20 antibody rituximab in patients with B cell non-Hodgkin's lymphoma: results of a prospective randomized controlled trial. Ann Hematol. 2009;88(1):51-57.
ClinicoEconomics and Outcomes Research

\section{Publish your work in this journal}

ClinicoEconomics \& Outcomes Research is an international, peerreviewed open-access journal focusing on Health Technology Assessment, Pharmacoeconomics and Outcomes Research in the areas of diagnosis, medical devices, and clinical, surgical and pharmacological intervention. The economic impact of health policy and health systems

\section{Dovepress}

organization also constitute important areas of coverage. The manuscript management system is completely online and includes a very quick and fair peer-review system, which is all easy to use. Visit http://www.dovepress.com/testimonials.php to read real quotes from published authors. 